\title{
Interfaz humano-computadora basada en señales de electrooculografía para personas con discapacidad motriz
}

Daniel Pacheco Bautista

Posgrado en Ingeniería Biomédica Universidad Popular Autónoma del Estado de Puebla (UPAEP).

daniel.pacheco01@upaep.edu.mx

Ignacio Algredo Badillo

Departamento de Ingeniería en computación Universidad del Istmo algredobadillo@sandunga.unistmo.edu.mx

David De la Rosa Mejía

Posgrado en Ingeniería Biomédica Universidad Popular Autónoma del Estado de Puebla (UPAEP). david.delarosa01@upaep.edu.mx

Aurelio Horacio Heredia Jiménez

Posgrado en Ingeniería Biomédica Universidad Popular Autónoma del Estado de Puebla (UPAEP). aureliohoracio.heredia@upaep.mx

Resumen: En este trabajo se presenta el desarrollo de un prototipo que asiste, a personas con cierta discapacidad motriz, en la interacción con la computadora de una forma simple y económica, mediante señales de electrooculografía. Esta técnica permite detectar los movimientos oculares basada en el registro de la diferencia de potencial existente entre la córnea y la retina, tal propiedad es aprovechada en este proyecto para controlar el desplazamiento del cursor 
del ratón de una forma precisa sobre la pantalla de la computadora. El prototipo es un diseño compacto alimentado de una fuente única de $5 \mathrm{~V}$ proveniente del puerto USB y utiliza la circuitería ya implementada en cualquier ratón electromecánico convencional con mínimas modificaciones. El uso de tales dispositivos así como de electrodos convencionales hace el producto de un costo relativamente bajo en relación a las propuestas en otros trabajos.

Palabras clave: Electrooculografía, discapacidad motriz, Interfaz humanocomputadora, Ingeniería biomédica.

\section{Human-computer interface based on signals of electrooculography for physical disabled people}

Abstract: In this paper, the development of a prototype is presented, which allows people with certain mobility disabilities interact with the computer. The design of the prototype is composed of different elements on a simple and economical way by mainly using electrooculographic signals. It is important to highlight that the proposed technique enables the detection of the eye movements based on the records of the potential difference between cornea and retina. In this project, this last property is used to control the movement of the mouse cursor on the computer screen in a precise way. The prototype is powered from a single 5V supply from USB port, uses compact circuitry and is implemented in a conventional electromechanical mouse with minimal modifications. The use of such devices as well as common electrodes make possible that the final product presents a low-cost proposal.

Keywords: Electrooculography, mobility disabilities, human-computer interface, biomedical engineering. 


\section{Introducción}

La discapacidad motriz es un padecimiento que en la actualidad se ha incrementado considerablemente, solo en México, según fuentes del Instituto Nacional de Estadística y Geografía (INEGI) alrededor del 5\% de la población presenta discapacidad de algún tipo, del cual el 58\% corresponde al tipo motriz. El origen y naturaleza de tales padecimientos son múltiples sin embargo en la mayoría de los casos la actividad mental y la funcionalidad de los ojos del individuo permanecen intactas, siendo este último un excelente recurso que puede permitir a la persona discapacitada seguir interactuando con su entorno realizando actividades de manera prácticamente normal.

Existen en la actualidad, diversos trabajos dedicados al desarrollo de prototipos que utilizan las señales eléctricas generadas en los ojos, obtenidas mediante electrooculografía (EOG), para controlar diferentes objetos tales como sillas de ruedas (Yathunanthan, Chandrasena, Umakanthan, Vasuki \& Munasinghe, 2008), (Arai \& Mardiyanto, 2011), brazos robotizados (Hao et al., 2013), interfaces gráficas de asistencia a discapacitados (Ding, Tong \& Li, 2005), cursor y teclado de la computadora (Rosas-Cholula, Ramírez Cortés, Escamilla Ambrosio \& Alarcón Aquino, 2011), (Caballero Gaviria, Castro Miller, Ordoñez Medina \& Rojas González, 2011), (Merino, Rivera, Gómez, Molina \& Dorronzoro, 2010), entre otros. En particular el problema del control del cursor mediante señales EOG ha sido tratado de diversas maneras, en (Rosas-Cholula, et al., 2011) los autores presentan un prototipo usando señales EOG obtenidas indirectamente mediante un set comercial inalámbrico de 16 electrodos, originalmente usado para adquisición de señales de electroencefalografía (ECG), la posición del cursor se obtiene a partir de la información de un giroscopio incluido en el set, mientras que los clics son generados a través del parpadeo voluntario del usuario mediante las señales EOG, para tal propósito ellos proponen la técnica de descomposición en modo empírico (EMD) como una herramienta computacional rápida y simple, para distinguir entre el parpadeo natural y los voluntarios para el control. Otro diseño importante se encuentra en 
(Caballero Gaviria, et al., 2011), en este los autores proponen una interface humano computadora basada en EOG consistente de cuatro etapas: adquisición de señales, filtros, amplificadores y un sistema de reconocimiento de patrones y generación de señales de control, el prototipo utiliza circuitos de señal mixta (PSoC), lo cual reduce el número de componentes del mismo, sin embargo solo está enfocado en los movimientos verticales y horizontales del cursor, sin atender a la funcionalidad del clic. En (Merino et al., 2010) se propone un algoritmo para el procesamiento de señales de EOG, el cual utiliza como entradas la derivada y el nivel de amplitud de la señal, la derivada es utilizada para detectar las orillas de las señales, y el nivel de amplitud para filtrar ruido, aun cuando el algoritmo muestra altas expectativas, el artículo no concluye con una implementación final.

En este trabajo se presenta el diseño de un dispositivo basado en señales de EOG para el control de las funcionalidades del mouse, siguiendo como objetivo además de la efectividad y robustez, el bajo costo del mismo. La implementación utiliza dispositivos de fácil adquisición y el algoritmo está basado en una simple máquina de estado implementada en un microcontrolador de gama media de Microchip. El resto de este artículo está organizado de la siguiente manera: La sección II describe el principio básico de la electrooculografía, la amplitud y ancho de banda de la señal, y las bases del funcionamiento de un ratón electromecánico. La sección III muestra el diseño del dispositivo, en la sección IV se presentan las pruebas y resultados obtenidos y finalmente en la sección $\mathrm{V}$ se mencionan las conclusiones y el trabajo a futuro. 


\section{Fundamento teórico}

\section{La electrooculografía}

El Electrooculograma (EOG) es un método de registro de los movimientos oculares basado en la detección de la diferencia de potencial existente entre la córnea y la retina. El origen de esta diferencia se encuentra en el epitelio pigmentario de la retina y permite considerar la presencia de un dipolo, donde la córnea corresponde al extremo positivo y la retina al extremo negativo, ver figura 1. Si el ojo se mueve desde la posición central directo a la periferia la retina se aproxima a uno de los electrodos, mientras que la córnea al electrodo del lado opuesto. Este cambio en la orientación del dipolo se refleja en un cambio en la amplitud y la polaridad de la señal EOG. Por consiguiente, resulta inmediato que el análisis de estos cambios permite determinar el movimiento de los ojos. La señal EOG puede obtenerse fácilmente mediante un par de electrodos conectados en la vecindad de los ojos más un electrodo de referencia como se muestra en la figura 2. De esta manera se tienen dos canales correspondiendo al movimiento horizontal y al movimiento vertical, respectivamente. De acuerdo a estudios previos, las señales EOG muestran amplitudes de señales que van de $5 \mathrm{uV}$ a $20 \mathrm{uV}$ por grado con un ancho de banda de $0 \mathrm{~Hz}$ a $30 \mathrm{~Hz}$ (Singh \& Singh, 2012), (Brown, Marmor, Vaegan, Brigell \& Bach, 2006). Una forma de onda idealizada EOG puede observarse en la figura 3.

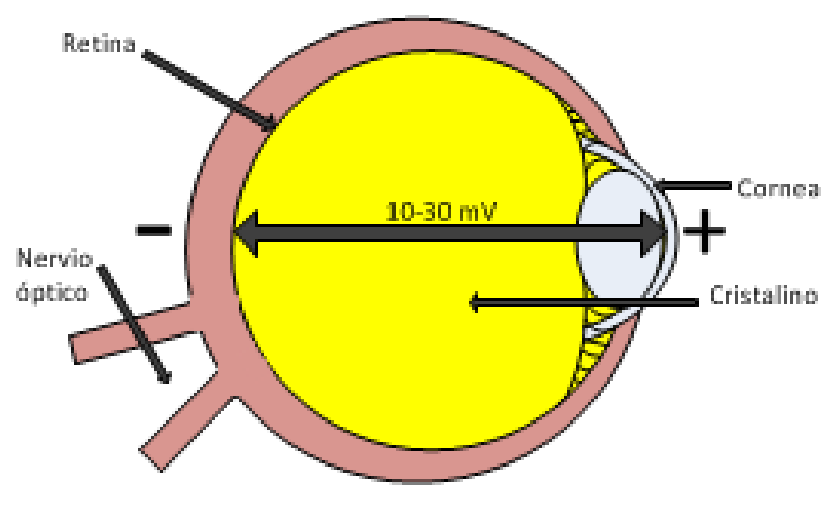

Figura 1. El ojo modelado como un dipolo. 


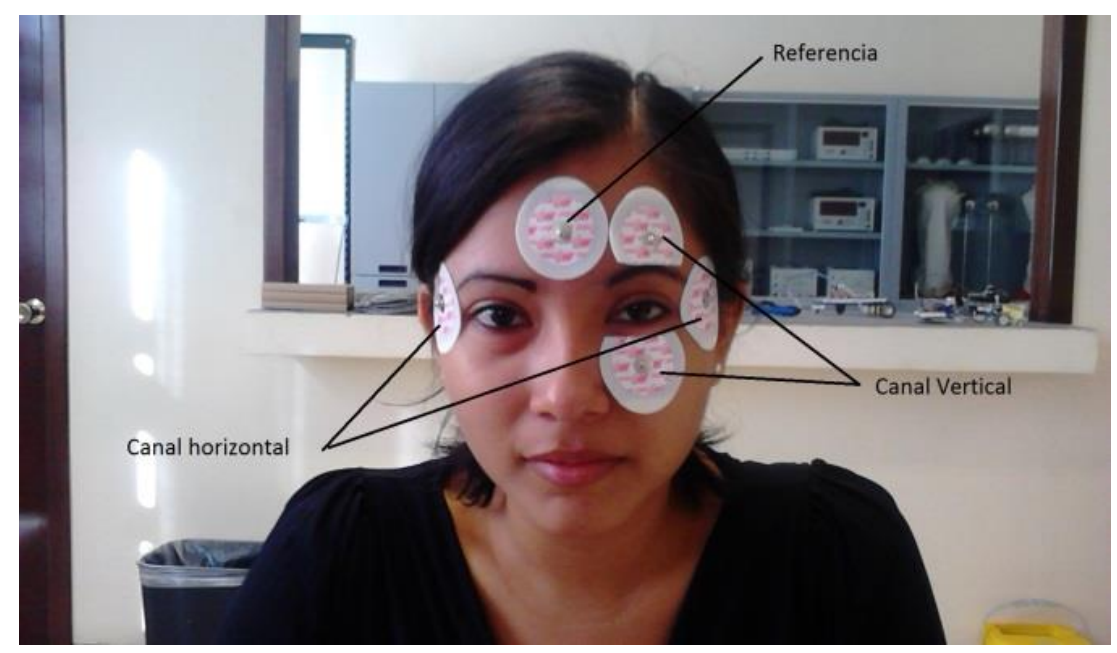

Figura 2. Ubicación de los electrodos.

Es posible detectar diversos movimientos del ojo a través del EOG, no obstante, en este trabajo se utilizan solo los tres principales: sacádicos, fijaciones y parpadeos (Bulling, Ward, Gellersen \& Troster, 2010), donde los sacádicos corresponden a los movimientos del ojo al cambiar rápidamente la mirada de un punto a otro (generalmente mayor a los 30 grados), las fijaciones resultan de mantener la mirada en un mismo punto y los parpadeos son los movimientos involuntarios y periódicos del ojo para humedecer la córnea y la conjuntiva. 


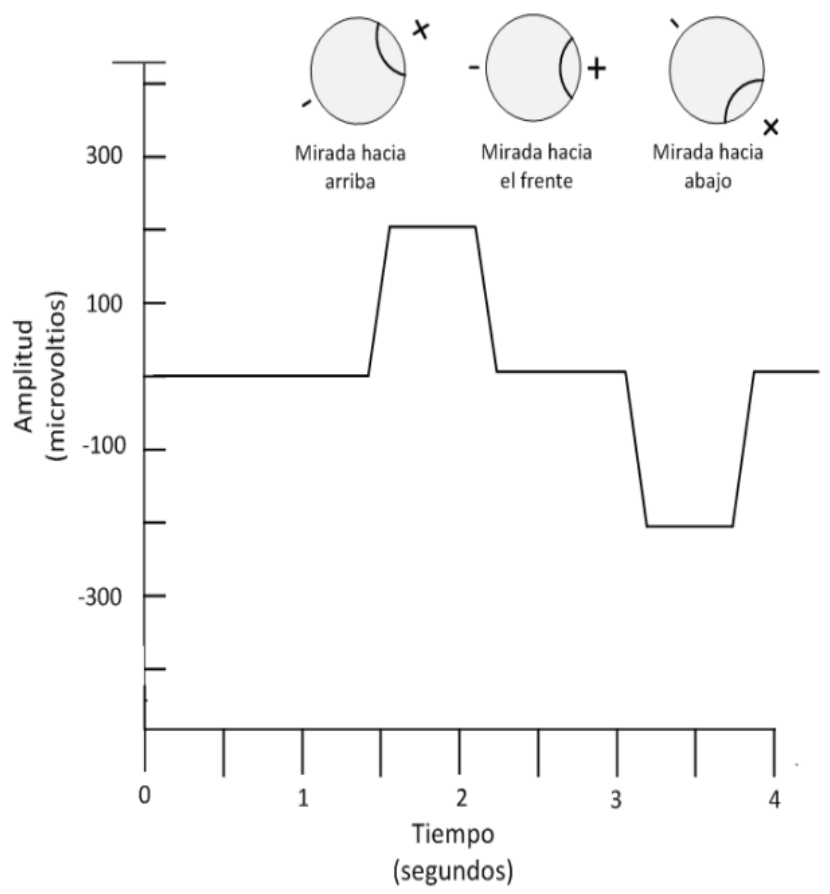

Figura 3. Forma de onda EOG ideales cuando la persona mira hacia arriba, al frente y hacia abajo.

\section{El ratón electromecánico USB}

El ratón electromecánico funciona a partir de una bola de caucho acoplada a tres rodillos giratorios: dos de arrastre y uno de apoyo como se muestra en la figura 4. El cilindro de apoyo o estabilizador se encarga de mantener la bola presionada contra los de arrastre, mientras que éstos están colocados perpendicularmente entre sí y giran cuando la bola se mueve, descomponiendo el desplazamiento en las componentes cartesianas $x$ e $y$ (horizontal y vertical). 


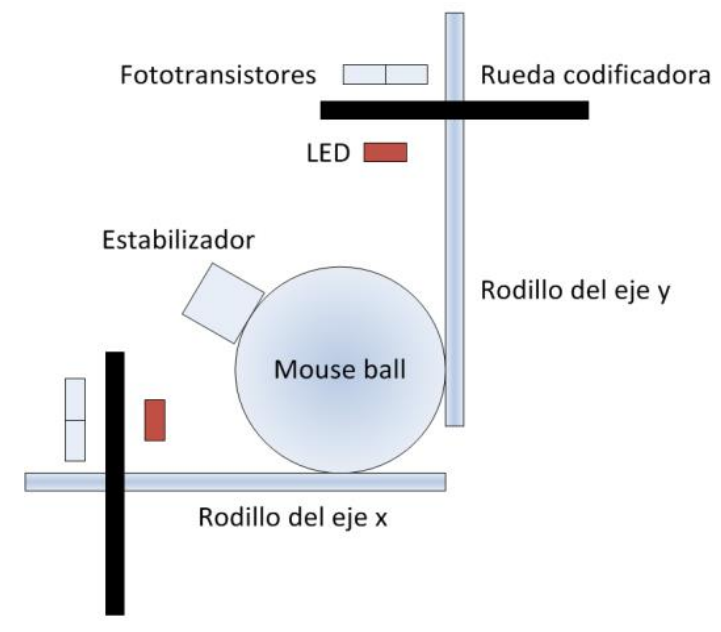

Figura 4. Sección mecánica del ratón.

Al extremo de cada cilindro de arrastre se encuentra un codificador, el cual consiste de una rueda con orificios, un emisor de luz en uno de sus lados y un detector de luz en el opuesto, ver figura 5. Cuando la rueda gira obligado por la bola, se producen interrupciones del haz de luz de manera intermitente. Por ejemplo, movimientos a la izquierda y a la derecha pueden verse como en la figura $6 a$ y $6 \mathrm{~b}$, respectivamente. La circuitería del ratón transforma esa información luminosa en movimientos, sumando el número de interrupciones de luz para evaluar cuánto ha de desplazarse y en qué dirección. Esta información descompuesta en $x$ e y se lleva al ordenador para indicarle al cursor cómo desplazarse.

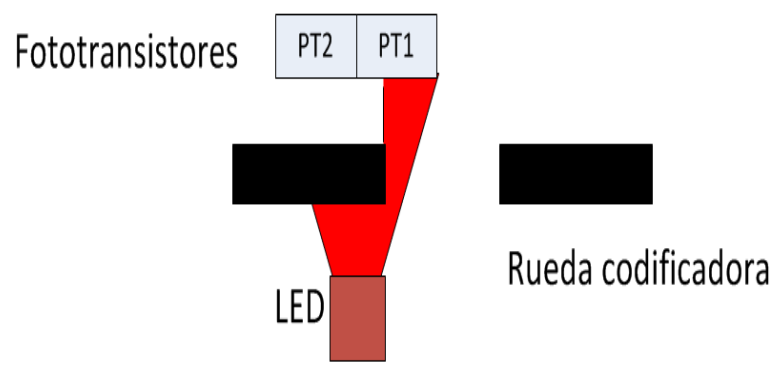

Figura 5. Sección optomecánica del ratón. 


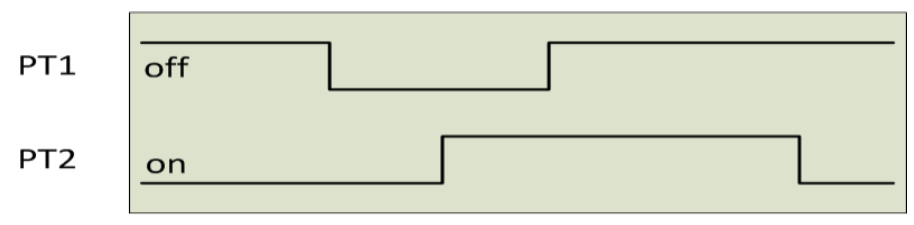

(a)

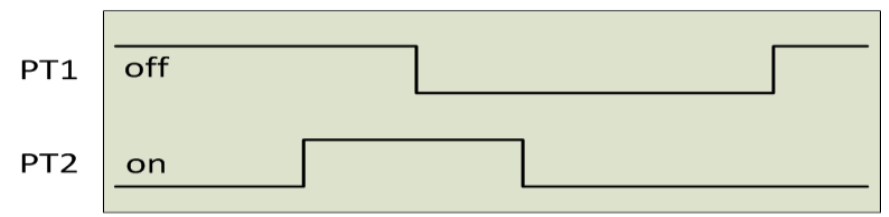

(b)

Figura 6. Salida del codificador en un a) movimiento a la izquierda y un b) movimiento a la derecha.

\section{Diseño del prototipo}

En la figura 7 se muestra el diagrama a bloques del dispositivo propuesto, el cual está formado de los siguientes bloques principales: 1) amplificación y filtrado, 2) conversión analógica digital (CAD) y procesamiento digital, 3) comunicación con la computadora, y fuente de alimentación. En esta sección se describen brevemente cada uno de estos bloques.

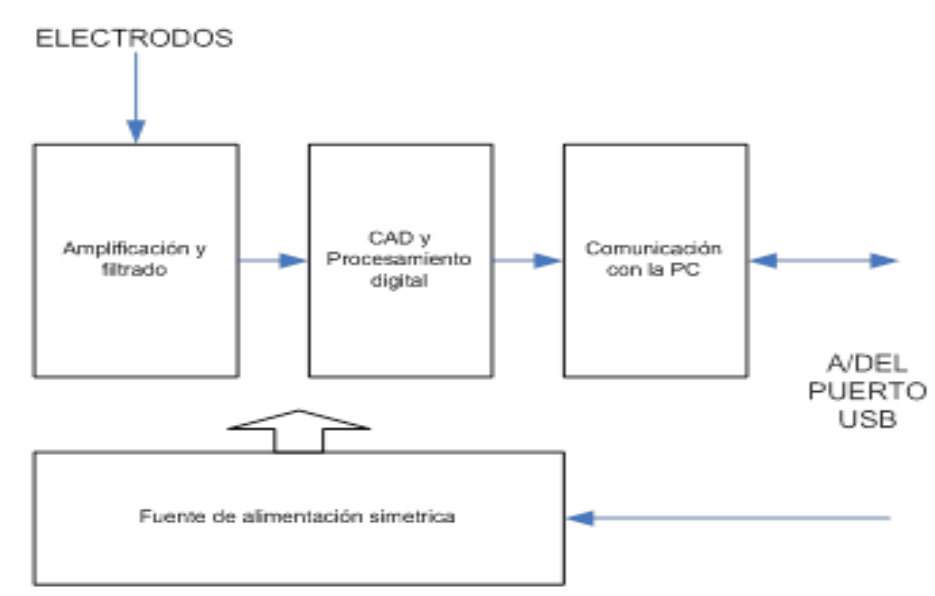

Figura 7. Diagrama a bloques del prototipo. 


\section{Amplificación y filtrado}

Las señales de EOG presentan amplitudes muy pequeñas del orden de $10 \mathrm{uV} \mathrm{a}$ $300 \mathrm{uV}$ con un ancho de banda de 0 a $30 \mathrm{~Hz}$ (Singh \& Singh, 2012) (Brown, et al., 2006), por consiguiente un amplificador de EOG además de presentar alta ganancia debe tener una excelente respuesta en bajas frecuencias. En particular en este diseño, se eligió una ganancia total de 4725 (proporcionada por dos amplificadores en cascada), cuya motivación principal es obtener en la salida señales con amplitudes que van de $47 \mathrm{mV}$ a $1.5 \mathrm{~V}$, las cuales resultan apropiadas para su conversión al formato digital en la siguiente etapa (CAD y procesamiento digital). Por otra parte, el ancho de banda de los filtros fue diseñado de $0.15 \mathrm{~Hz}$ a $30 \mathrm{~Hz}$, resaltando que la discriminación de algunas frecuencias del espectro no resultan críticas debido a la naturaleza de la aplicación y fue necesario para disminuir el efecto de línea base a la deriva, como se explica posteriormente. Adicionalmente, existe una gran cantidad de ruido e interferencia que corrompe la señal deseada y la hace difícil de distinguir, tales como el ruido de la línea de distribución de energía, la interferencia electromagnética, el ruido ocasionado por otros dispositivos electrónicos, los artefactos de movimiento que cambian la interfaz piel-electrodo, la contracción de los músculos o electromiografía, la respiración, entre otros, muchas de estas señales suelen manifestarse en modo común por lo que una elevada razón de rechazo en modo común (CMRR) es otra característica deseable del diseño (Choudhury, Venkataramanan, Nemade \& Sahambi, 2005), (Acharya , 2011).

El bloque de amplificación y filtrado es idéntica para ambos canales horizontal y vertical, por lo que en lo que sigue se hace referencia a sólo uno de estos. El diseño y desarrollo de este bloque fue dividido en tres secciones, donde la primera se muestra en la figura 8. El núcleo de esta primera sección es el amplificador de instrumentación INA114, el cual reporta una elevada CMRR de $115 \mathrm{~dB}$, el cual es un valor apropiado para este tipo de aplicaciones (Choudhury, et al., 2005) y fue configurado para proporcionar una ganancia $G$ de 225, a partir de la ecuación 1, donde Rg es la resistencia equivalente de R1, R4 y RV2. 


$$
G=1+\frac{50 k}{R g}
$$

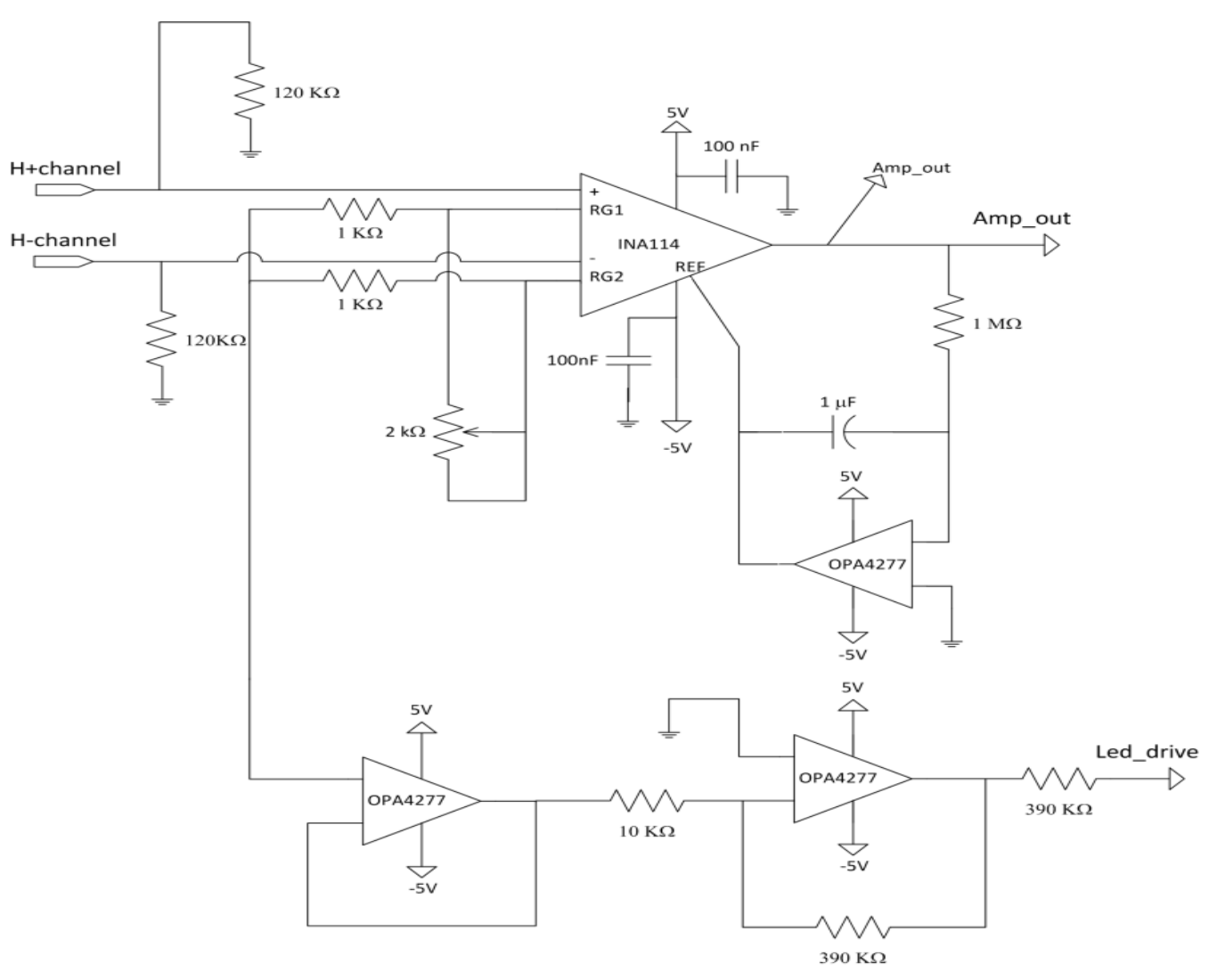

Figura 8. Primera sección de la etapa de amplificación y filtrado: Amplificación, Cancelación de offset e incremento de la CMRR.

Algunas señales de interferencia y la polarización de los electrodos suelen combinarse creando un efecto conocido como línea base a la deriva, es decir la línea de referencia de la señal de salida se mueve lentamente hacia una de las fuentes de polarización sin razón aparente, lo cual provoca la saturación del amplificador en un periodo corto de tiempo. Para evitar este efecto, un amplificador operacional (OPA4277 mostrado en la parte media de la figura 8) integra la componente en DC de la salida, la invierte y la realimenta al punto de referencia. Tal restaurador de DC cambia el amplificador original acoplado en DC a un amplificador con acoplo en AC, donde la frecuencia de corte del mismo es de $0.15 \mathrm{~Hz}$, calculado a partir de la ecuación 2 . 


$$
f_{-3 d B}=\frac{1}{2 \pi R_{3} C_{1}}
$$

Adicionalmente para mejorar el rechazo al ruido en modo común, dos amplificadores operacionales (OPA4277 mostrados en la parte inferior de la figura 8) invierten y realimentan el voltaje en modo común de la entrada al cuerpo del paciente, además, en caso de una condición donde el paciente accidentalmente sea conectado a la tierra del sistema, éste provee la trayectoria para la corriente desde el nodo Vref (Acharya , 2011).

La segunda sección, mostrada en la figura 9, es un amplificador inversor convencional, el cual proporciona una ganancia adicional de 21 , al mismo tiempo que desplaza la línea base a 2 volts. Lo último es necesario para procesar la señal mediante el convertidor analógico digital del microcontrolador, lo cual se detalla posteriormente.

Finalmente la tercera sección es un filtro pasabajas activo, el cual está sintonizado a una frecuencia de corte de $30 \mathrm{~Hz}$, utilizando la configuración Sallen Key, ver figura 10. En este circuito la frecuencia de corte es calculada a partir de la ecuación 3.

$$
f_{-3 d B}=\frac{1}{2 \pi R_{20} \sqrt{\left(C_{8}+C_{9}\right) C_{10}}}
$$




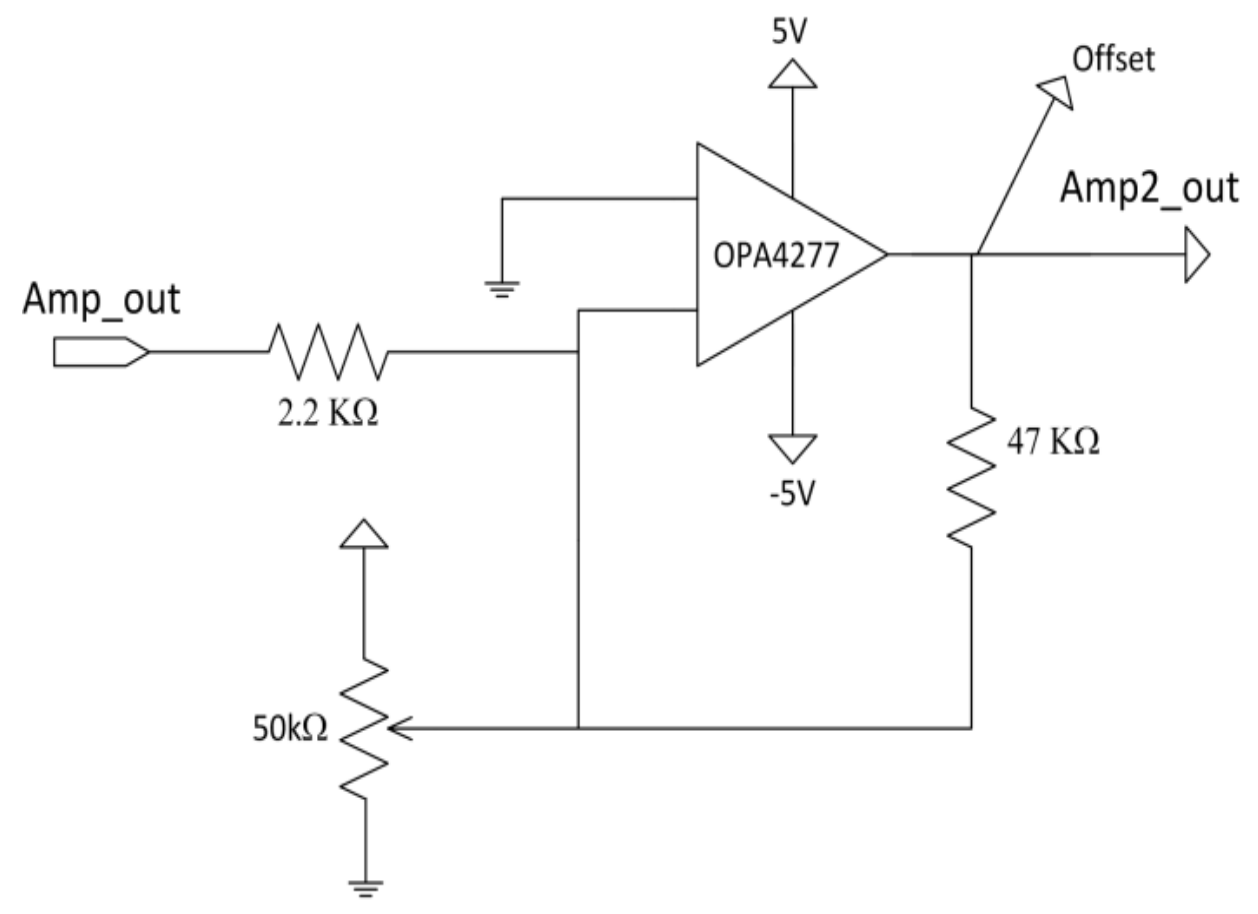

Figura 9. Segunda sección de la etapa de amplificación y filtrado: Amplificación y acondicionamiento de la señal.

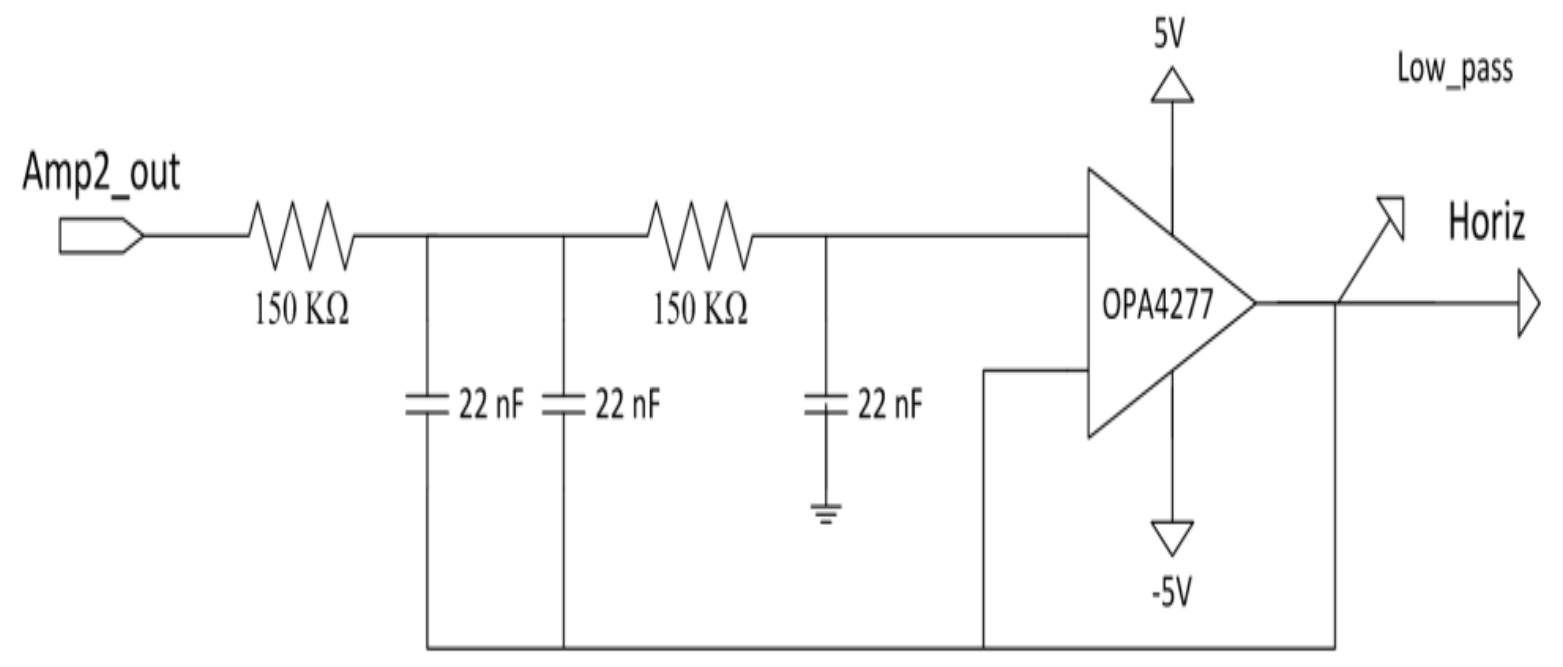

Figura 10. Tercera sección de la etapa de amplificación y filtrado: Filtro pasabajas. 


\section{CAD y Procesamiento digital}

Este bloque comprende la conversión analógica-digital y el procesamiento digital, ambas funciones fueron implementadas en el microcontrolador PIC16F618. El uso de tal dispositivo y, en particular, de su convertidor analógicodigital, es de manera directa, por lo que en esta sección únicamente se explicará la lógica usada para generar las señales a enviar a la etapa de comunicación a partir de las señales previamente amplificadas y filtradas. Inicialmente, se desarrolló un simple protocolo para el uso del dispositivo, el cual consiste de los siguientes cuatro pasos:

1. El usuario se ubicará a una distancia de 30 a $40 \mathrm{~cm}$ de la pantalla, observando al punto central de la misma.

2. Un movimiento sacádico a la izquierda iniciará el recorrido del cursor hacia la izquierda. En este momento el usuario puede regresar la mirada a cualquier otro punto de la pantalla.

3. Un movimiento sacádico a la derecha detendrá automáticamente el cursor.

4. En ese instante, si el usuario así lo desea, un guiño voluntario del ojo seleccionará el objeto señalado.

Situaciones duales ocurren cuando se desea mover el cursor en dirección contraria, hacia arriba o hacia abajo de la pantalla. Es importante resaltar que los movimientos lentos del ojo y con ángulos menores a 30 grados (es decir, movimientos no sacádicos) no afectan el protocolo, lo que evita que el usuario genere movimientos indeseables del cursor al observar algún icono o menú en la pantalla. De igual forma los guiños involuntarios para humectar el ojo resultan despreciables debido a su pequeña magnitud y duración.

Se seleccionó este protocolo buscando que el usuario realice la mínima cantidad de movimientos en los ojos, evitando de esta manera su cansancio. Al mismo tiempo, un protocolo sencillo proporciona robustez y simplicidad al sistema. Es importante notar que para el uso de este esquema es suficiente con detectar la existencia de un movimiento sacádico o la ausencia de éste, sin la preocupación en la duración o la magnitud exacta del mismo. A partir de las formas de onda 
de la figura 11, el protocolo fue implementado mediante la máquina de estados mostrada en la figura 12, donde un pulso positivo corresponde a un movimiento sacádico a la izquierda (o hacia arriba), mientras que un pulso negativo es generado por un movimiento sacádico a la derecha (o hacia abajo). Observe como en esta grafica los pulsos ya no son perfectamente rectangulares como en la Figura 3, lo que modela el acoplo en AC resultante de agregar el integrador en la salida del amplificador diferencial. Las etiquetas N1, P1, N2 y P2 son niveles de umbral, necesarios para la operación de la máquina de estados de la Figura 12.

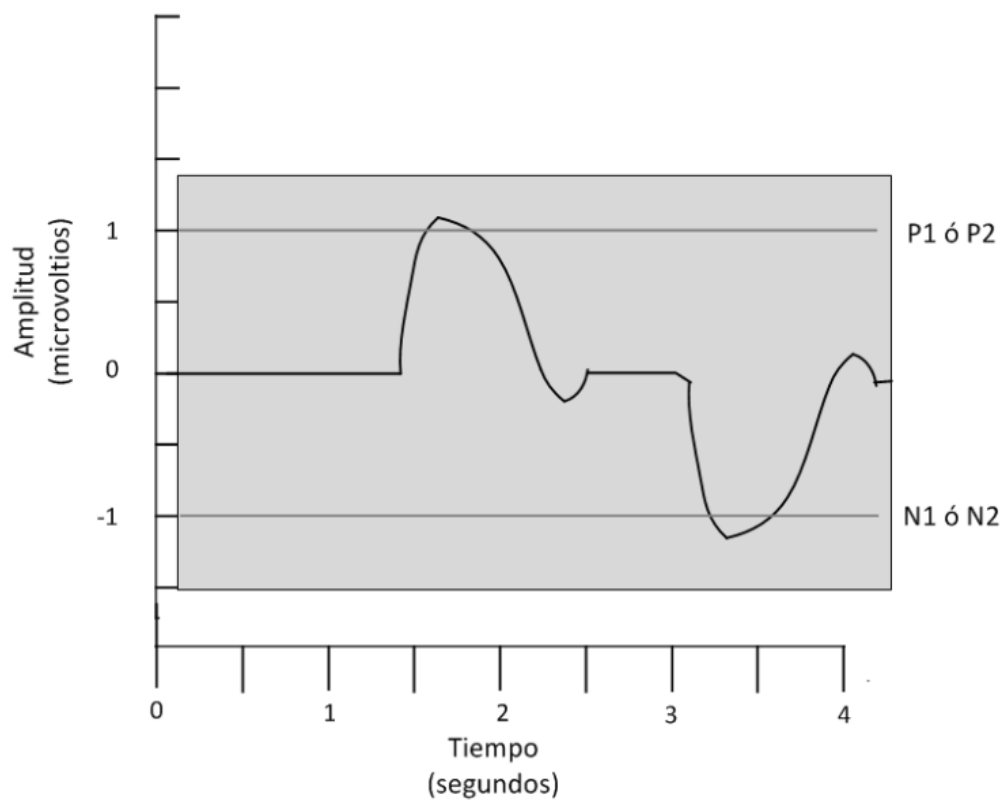

Figura 11. Definición de los niveles de umbral. 


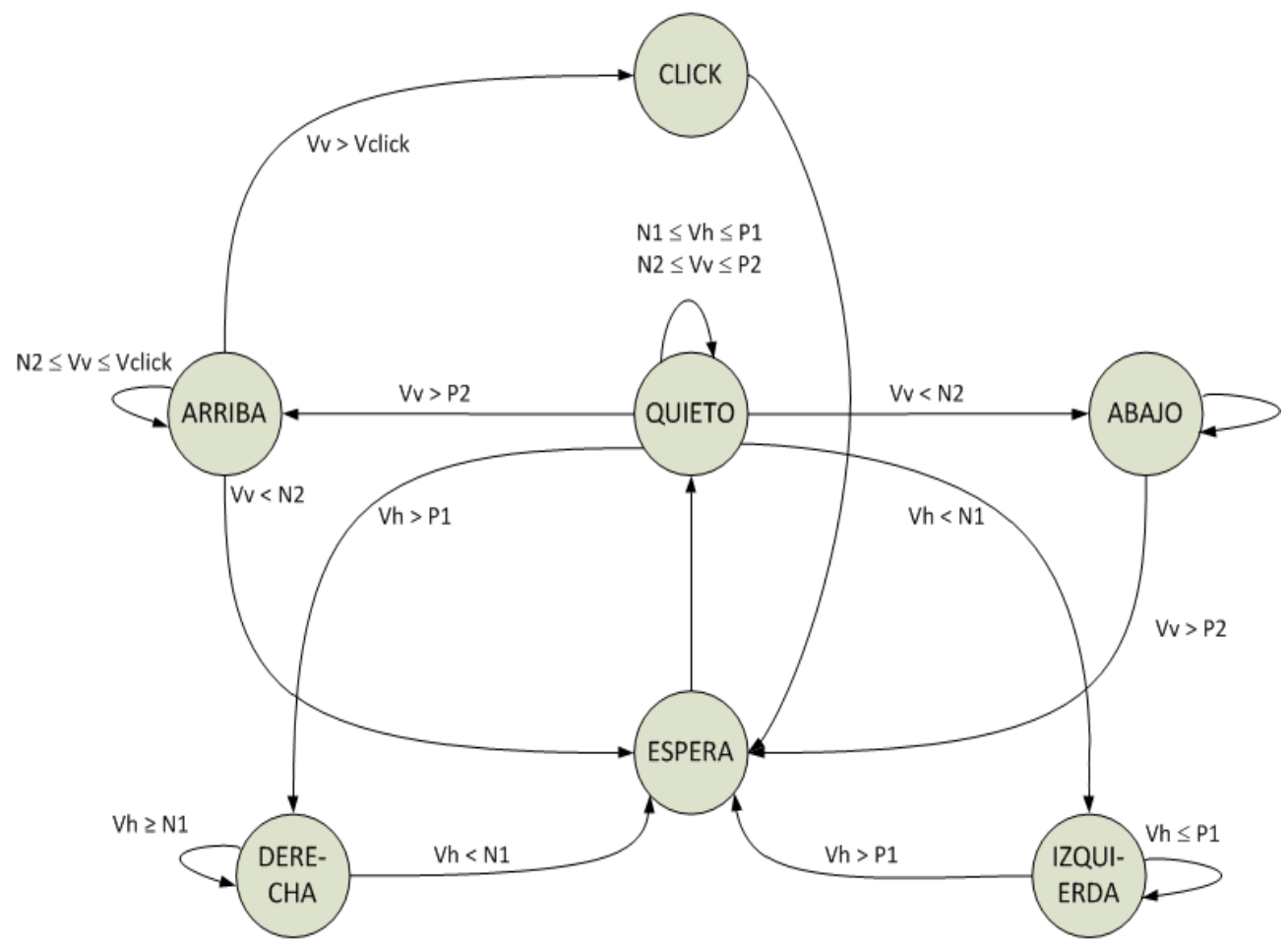

Figura 12. Máquina de estados para implementar el protocolo.

En la figura 11, las variables $V_{v}$ y $V h$ representan la salida del convertidor analógico-digital de los canales vertical y horizontal, respectivamente. Los estados derecha e izquierda, generan fundamentalmente las secuencias mostrada en la figura $6 a$ y $6 \mathrm{~b}$ para el canal horizontal, mientras que los estados arriba y abajo generan esas mismas secuencias para el canal vertical. El estado click genera un pulso cuadrado en el Pin RB7 del microcontrolador, mientras que el estado delay únicamente es un ciclo de retardo de $300 \mathrm{~ms}$.

Partiendo del estado quieto, si el usuario no genera ningún movimiento sacádico, Vv yVh no superarán en magnitud los umbrales P2 y N2 y P1 y N1 respectivamente, permaneciendo en ese mismo estado. Si por ejemplo, estando en esta condición el usuario mira rápidamente hacia la derecha, Vh superara a P1 y la máquina adquiere el estado derecha, generando las señales que iniciaran el recorrido del cursor hacia esa misma dirección. En estos momentos un movimiento de la mirada hacia la izquierda provocará que Vh sea menor a N1, 
pasando al estado espera, el cual básicamente es un retardo utilizado para ignorar la parte complementario del segundo pulso. La secuencia es similar para los otros movimientos del cursor. El clic fue modelado por un guiño fuerte del ojo el cual es un pulso positivo muy similar al de un movimiento sacádico hacia arriba, pero de mayor magnitud y menor duración, al ocurrir tal movimiento, la máquina de estados adquiere el estado click, en el cual se genera básicamente un pulso de 300ms en el Pin RB7.

\section{Comunicación con la PC}

Los pines del microcontrolador correspondientes a las secuencias generadas por la etapa previa para el movimiento horizontal y vertical, así como el pulso que emula el clic, son conectados al circuito de un ratón electromecánico, permitiendo que este realice la comunicación con el PC. Para tal efecto los fototransistores y el interruptor del clic en el ratón original fueron retirados del circuito, conectando las señales de movimiento en los puntos correspondientes a los emisores de dichos fototransistores y el pulso que emula el clic en uno de los puntos donde fue retirado el interruptor.

\section{Fuente de alimentación simétrica}

La alimentación del circuito es a través del puerto USB de la misma computadora, no obstante la mayoría de los integrados operan con fuentes simétricas, por consiguiente fue necesario incluir una fuente de voltajes simétricos de $\pm 5 \mathrm{~V}$ a partir del integrado TC7660CPA, el cual basa su operación en la conmutación de capacitores. El diagrama de conexión es mostrado en la figura 13. 


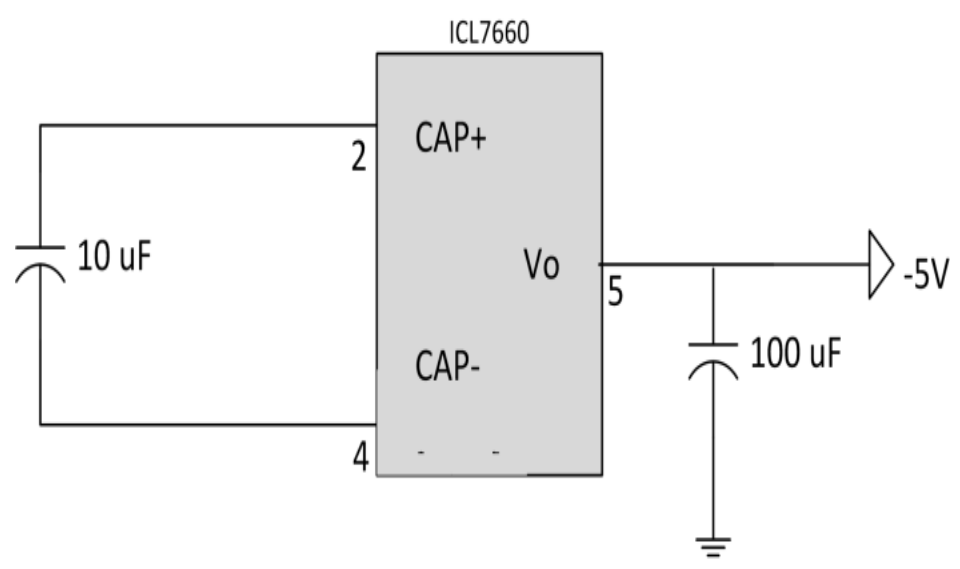

Figura 13. Fuente de alimentación simétrica.

\section{Resultados obtenidos}

El circuito fue simulado en el software de desarrollo Proteus v7.6 y posteriormente implementado completamente, teniendo un consumo de potencia de aproximadamente $450 \mathrm{~mW}$, sin considerar la etapa de comunicación con la computadora. La respuesta en frecuencia de la etapa de amplificación y filtrado simulada se muestra en la figura 14 y se muestra que el ancho de banda y la amplitud de la señal son acorde con lo calculado $(0.15 \mathrm{~Hz}-30 \mathrm{~Hz}$ y 4725 , respectivamente). En la figura 15 puede apreciarse la forma de onda obtenida al realizar el usuario un movimiento sacádico a la derecha seguido de uno a la izquierda, en tal imagen se puede observar una señal estable, de suficiente amplitud (800 mV aproximadamente) y con el problema de línea base eliminado. Tales mediciones fueron realizadas con el osciloscopio digital Tektronix TDS2022. 


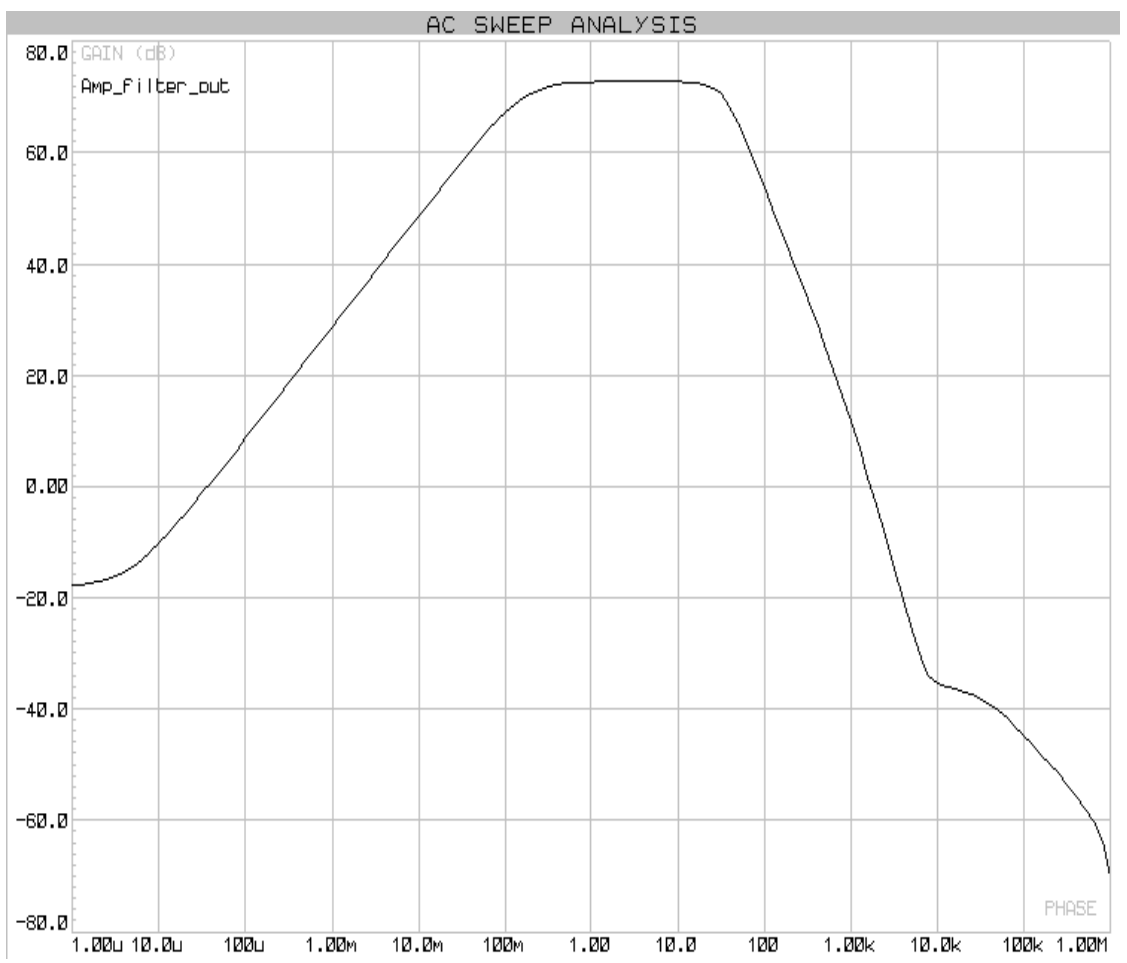

Figura 14. Respuesta en frecuencia de la etapa de amplificación y filtrado.

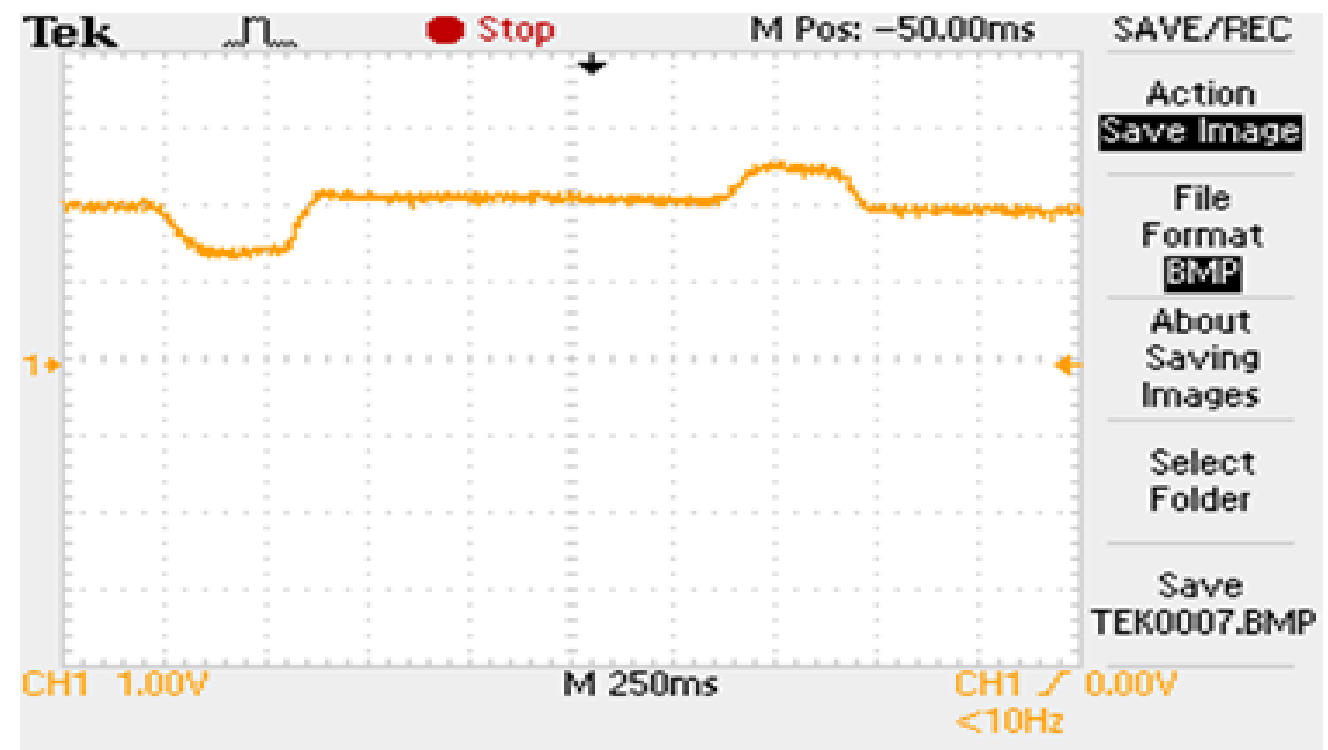

Figura 15. Forma de onda obtenida al realizar un movimiento sacádico a la derecha y luego uno a la izquierda. 
Finalmente, la funcionalidad del dispositivo fue evaluado en 20 personas. Las pruebas se dividieron en dos grupos como muestra la tabla 1, donde el primer grupo consiste en inicializar un movimiento del cursor (en cada una de las 4 direcciones) para posteriormente detener su recorrido, comenzando en posiciones aleatorias de la pantalla. En el segundo grupo y partiendo del centro de la pantalla, el usuario tenía como objetivo ubicar el cursor sobre un icono localizado en la parte superior derecha de la misma y, posteriormente, seleccionarlo mediante un clic, en donde la trayectoria que el cursor debería seguir fue libre. Como puede observarse en la tabla 1, el dispositivo opera apropiadamente en el $100 \%$ de los casos de movimiento y detención sin embargo aún muestra problemas en la generación del clic, lo cual se atribuye a diferencias en la amplitud y duración del pulso entre los usuarios.

\begin{tabular}{|l|l|l|}
\hline CLASIFICACION & PRUEBAS & PERSONAS CON EXITO \\
\hline \multirow{3}{*}{ Grupo 1 } & Iniciar y detener movimiento a la izquierda & 20 \\
\cline { 2 - 3 } & Iniciar y detener movimiento a la derecha & 20 \\
\cline { 2 - 3 } & Iniciar y detener movimiento hacia arriba & 20 \\
\cline { 2 - 3 } & Iniciar y detener movimiento hacia abajo & 20 \\
\hline \multirow{2}{*}{ Grupo 2 } & Partir del centro de la pantalla y posicionarse en el icono & 20 \\
\cline { 2 - 3 } & Seleccionar posteriormente el icono & 12 \\
\hline
\end{tabular}

Tabla 1. Resultados de las pruebas realizadas en 20 usuarios.

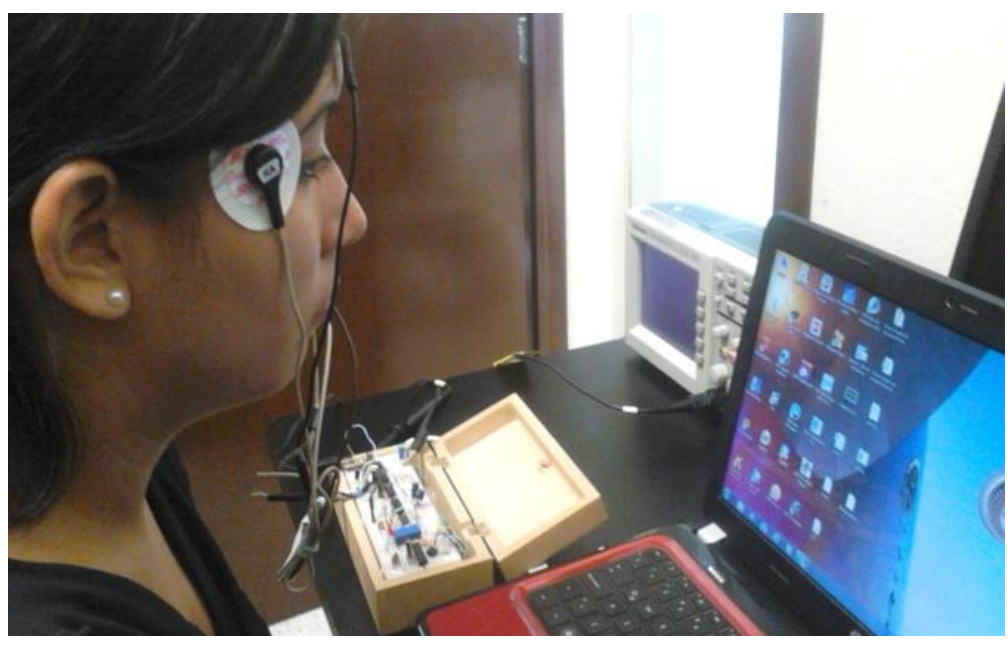

Figura 16. Prototipo siendo probado por un usuario. 


\section{Conclusiones}

Se ha realizado un dispositivo de interfaz humano computadora para personas con discapacidad motriz, a través de señales de electrooculografia, cuyo diseño es compacto, eficiente, robusto y fácil de utilizar. Siendo además de bajo consumo de potencia y económico en relación a otras propuestas en el mercado. En lo sucesivo se pretende optimizar la velocidad de la interfaz y eliminar la limitante encontrada al realizar el clic sobre la pantalla. También se pretende incluir toda la lógica de comunicación, actualmente en la circuitería del ratón electromecánico, en el mismo microcontrolador. El proyecto muestra cómo la electrooculografia es una alternativa muy eficiente y económica para el control de dispositivos. Este mismo proyecto podría utilizarse en lo sucesivo en el control de sillas de ruedas o robots de asistencia, sólo por mencionar algunos ejemplos.

\section{Reconocimientos}

Este proyecto fue apoyado por el Programa para el mejoramiento del profesorado (PROMEP) y la Universidad del Istmo (UNISTMO), a través de la beca para estudios de doctorado con folio UNISTMO-003. 


\section{Referencias}

Acharya V. (2011). Improving Common-Mode Rejection Using the Right-Leg Drive Amplifier. (Reporte de aplicación SBAA188), Texas Instruments.

Arai, K. \& Mardiyanto, R. (2011). Eyes based electric wheel chair control system. International journal of advanced computer science and applications, Vol 2 (12), pp. 98 105.

Brown, M., Marmor, M., Vaegan, Zrenner, E., Brigell, M. \& Bach, M. (2006). ISCEV Standard for Clinical Electro-oculography EOG. Documenta Ophthalmologica, Vol. 113 (3), pp. 205212.

Bulling, A., Ward, J. A., Gellersen, H. \& Troster, G. (2010). Eye movement analysis for activity recognition using electrooculography. IEEE transactions on pattern analysis and machine intelligence. Vol. 33(4). pp. 741-743.

Caballero Gaviria, A.D., Castro Miller, I.D., Ordoñez Medina, S \& Rojas González, D.M (2011) Implementación de una interfaz hombre-computador basada en registros EOG mediante circuitos de señal mixta PSoC. Proceeding of the V Latin American Congress on biomedical engineering, CLAIB 2011, La Habana, Cuba, pp. 16-21.

Choudhury, S. R., Venkataramanan, S., Nemade, H. B. \& Sahambi, J. S. (2005). Design and Development of a Novel EOG Biopotential Amplifier. International Journal of Business and Emerging Markets, Vol. 7(5), pp. 271-274.

Ding, Q., Tong, K. \& Li, G. (2005). Development of an EOG (Electro-Oculography) based human-computer interface, Proceedings of the 2005 IEEE Engineering in medicine and biology 27th annual conference, Shanghai, China, pp. 6829-6831.

Hao, Y., Controzzi, M., Cipriani, C., Popovic, D. B., Yang, X., Chen, W., Zheng, X. \& Chiara Carrozza M. (2013). Controlling Hand-Assistive Devices: Utilizing Electrooculography as a Substitute for Vision. Robotics \& Automation Magazine, IEEE. Vol. 20(1), pp. 40-52.

Merino, M., Rivera, O., Gómez, I., Molina, A. \& Dorronzoro, E. (2010). A Method of EOG Signal Processing to Detect the Direction of Eye Movements. First International Conference on Sensor Device Technologies and Applications, Venice/Mestre, Italy, pp. 100-105.

Rosas-Cholula, G., Ramírez Cortés, J.M., Escamilla Ambrosio, J. \& Alarcón Aquino V. (2011). On the development of a simple EOG-based mouse with $\mathrm{BCl}$ technology applying empirical mode decomposition and DWT. Proceeding of the 15th International graphonomics society Conference, IGS2011, Cancún, México. pp. 70-73.

Singh, H. \& Singh, J. (2012). A review on electrooculography. International Journal of Advanced Engineering Technology, Vol. 3(4), pp.112-122.

Yathunanthan, S., Chandrasena, L.U.R., Umakanthan, A., Vasuki, V. \& Munasinghe, S.R. (2008). Controlling a Wheelchair by Use of EOG Signal. Proceeding of 4th International Conference on Information and Automation for Sustainability, ICIAFS 2008. Colombo, Sri Lanka. 


\section{Notas biográficas:}

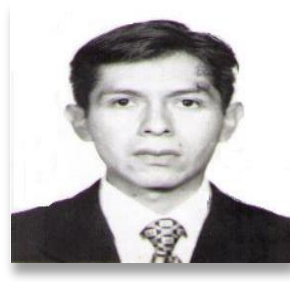

Daniel Pacheco Bautista. Recibió el grado de ingeniero en electrónica del Instituto Tecnológico de Oaxaca (ITO), en el 2000, y el grado de Maestro en ciencias con especialidad en diseño de circuitos integrados, del Instituto nacional de Astrofísica, Óptica y Electrónica (INAOE), en el 2003. Actualmente es estudiante del programa de doctorado en Ingeniería Biomédica, en la Universidad Popular Autónoma del Estado de Puebla (UPAEP), y profesor investigador en la Universidad del Istmo (UNISTMO). Sus líneas de investigación incluyen: Arquitectura de computadoras y Lógica reconfigurable, Osciladores controlados por voltaje y Circuito de amarre de fase en VLSI, y recientemente plataformas alternativas para el ensamble de secuencias cortas de ADN.

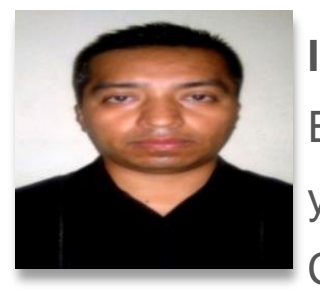

Ignacio Algredo Badillo. recibió el grado en Ingeniería Electrónica del Instituto Tecnológico de Puebla (ITP) en el 2002 y los grados de Maestría y Doctorado en Ciencias Computacionales del Instituto Nacional de Astrofísica, Óptica y Electrónica (INAOE) en el 2004 y 2008, respectivamente. Desde 2009, se desempeña como profesor-investigador de tiempo completo adscrito a la carrera de Ingeniería en Computación en la Universidad del Istmo. Él es miembro del SNI desde el 2011 y sus líneas de investigación son enfocadas en el diseño y desarrollo de sistemas digitales, arquitecturas reconfigurables, plataformas basadas en el concepto radio software, seguridad, prototipos didácticos, sistemas criptográficos, implementaciones en FPGA, sistemas basados en microcontroladores y microprocesadores y aceleración en hardware para aplicaciones específicas. 


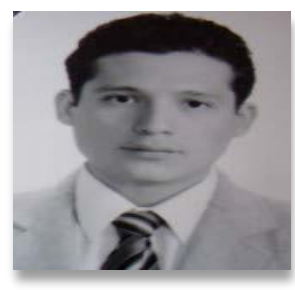

David De La Rosa Mejía. Nació en Tapachula Chiapas, el 18 de Septiembre de 1988, se graduó como Ingeniero biónico y actualmente estudiante de la Maestría en Ingeniería Biomédica en la Universidad Popular Autónoma del Estado de Puebla. Se ha desempeñado en el área Biomédica en mantenimiento preventivo y correctivo de instrumental Médico, actualmente trabaja en diseño y construcción de equipo Médico.

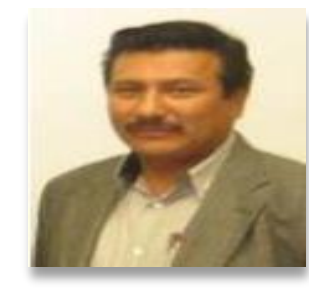

Aurelio Horacio Heredia Jiménez. Recibió el grado de ingeniero eléctrico de la Universidad Juárez Autónoma de Tabasco (UJAT), y los grados de Maestría y Doctorado con especial en óptica, del Instituto nacional de Astrofísica, Óptica y Electrónica (INAOE). Actualmente es profesor investigador en la Universidad Popular Autónoma del Estado de Puebla, adscrito al departamento de posgrado en Ingeniería Biomédica, siendo sus principales líneas de investigación: Diseño y construcción de equipo biomédico, diseño de microsistemas (MEMS/NEMS), Microcomponentes ópticos, y diseño de sistemas utilizando fibras ópticas y guías de onda.

\author{
(c) (i) (2) (2) \\ Esta obra está bajo una licencia de Creative Commons \\ Reconocimiento-NoComercial-Compartirlgual 2.5 México
}

